\title{
Analysis of shot noise suppression in mesoscopic cavities in a magnetic field
}

\author{
P. Marconcini, M. Macucci, G. Iannaccone, B. Pellegrini, and G. Marola \\ Dipartimento di Ingegneria dell'Informazione, Università di Pisa \\ Via Caruso, I-56122 Pisa, Italy
}

(Dated: November 30, 2018)

\begin{abstract}
We present a numerical investigation of shot noise suppression in mesoscopic cavities and an intuitive semiclassical explanation of the behavior observed in the presence of an orthogonal magnetic field. In particular, we conclude that the decrease of shot noise for increasing magnetic field is the result of the interplay between the diameter of classical cyclotron orbits and the width of the apertures defining the cavity. Good agreement with published experimental results is obtained, without the need of introducing fitting parameters.

PACS numbers: 72.70.+m, 73.23.-b, 75.75.+a
\end{abstract}

In the recent literature, the topic of shot noise suppression in mesoscopic structures has received significant attention, as a result of the formulation of several theoretical predictions 1, 2, 3, 4, 5] and the subsequent experimental confirmation [6, 7] of some of such predictions. All of the shot noise suppression phenomena are the result of correlations between charge carriers that decrease the variance of the random process corresponding to the elementary charges crossing the device: such correlations may result either from Fermi exclusion or from Coulomb interaction, and for their investigation powerful theoretical methods have been developed, ranging from Random Matrix Theory [8] to semiclassical techniques [9]. The convergence, in terms of shot noise suppression, between the results of quantum and semiclassical approaches has been explained, using a voltage probe technique, by van Langen and Büttiker [10], who have shown that dephasing phenomena do not have effects on the noise power spectral density. This result appears to be valid also in the presence of non DC bias 11. A detailed discussion of the fundamentals of shot noise in mesoscopic conductors can be found in ref. 12].

Particular interest has been raised by the so-called "chaotic cavities", mesoscopic regions delimited by input and output constrictions that are much smaller than the cavities themselves. Jalabert et al. [2] showed that in the case of symmetric apertures noise is suppressed down to $1 / 4$ of its full shot value: this theory received experimental confirmation [7] in 2001, and, more recently, the noise behavior of a chaotic cavity in an orthogonal magnetic field has been measured [13], observing a somewhat linear reduction of the Fano factor as the magnetic field is increased. In ref. 13] the authors motivate the decrease of the Fano factor as the magnetic field is increased with the reduction of the portion of the cavity area explored by the electrons, and rely on a fitting parameter, the quantum scattering time, which is also used to explain the behavior of the Fano factor with no magnetic field as the apertures are made wider. They also attribute the differences between the variation of the conductance and that of the Fano factor to a transition between "quantum" and "classical" chaos.

We provide an alternative, intuitive interpretation of the behavior in the presence of a magnetic field, based on the comparison between the classical cyclotron diameter and the constriction width, instead of the cavity dimensions, which is supported by the results of the numerical simulations that we present in the following. Furthermore, we argue that there is no significant contribution of "classical" chaos to the observed phenomena, in particular we suggest a different explanation of the preserved additivity of the constriction resistances up to 40 propagating modes, in the case of no magnetic field. Such an explanation is based on assuming some degree of nonideality in the experiment, specifically partial thermalization to the lattice temperature of the electron gas in the cavity.

In most of our calculations, we consider model cavities defined by hard walls and with a rectangular shape (see inset of fig. 1), in order to keep the computational time within reasonable limits, running a few checks for structures with different geometries: only in few particular cases small differences were observed, which will be detailed in the discussion of the results. We wish to point out that a classically chaotic shape is not needed to achieve the known results for shot noise suppression. This conclusion cannot be found in unequivocal and explicit form in the existing literature, except for the very recent paper by Aigner et al. 14], who have shown that the distribution of transmission eigenvalues (and therefore the Fano factor) in a cavity with narrow constrictions does not depend appreciably on its shape.

However, the theoretical basis needed to reach such a conclusion had already been developed by several authors. For example, we can apply to a single cavity, in the absence of a magnetic field, the analytic procedure discussed by Oberholzer et al. 15] for a series of cavities. The cavity is supposed to act as an elastic quasi-reservoir, effectively decoupling the two constrictions: by balancing the incoming and outgoing electron fluxes at each energy [15], one obtains, in the hypothesis of symmetric and narrow constrictions, the well-known result of $1 / 4$ for the Fano factor.

We wish to point out that to arrive at this result, no hypothesis of a classically chaotic shape is needed: the discontinuities represented by the transitions between the 

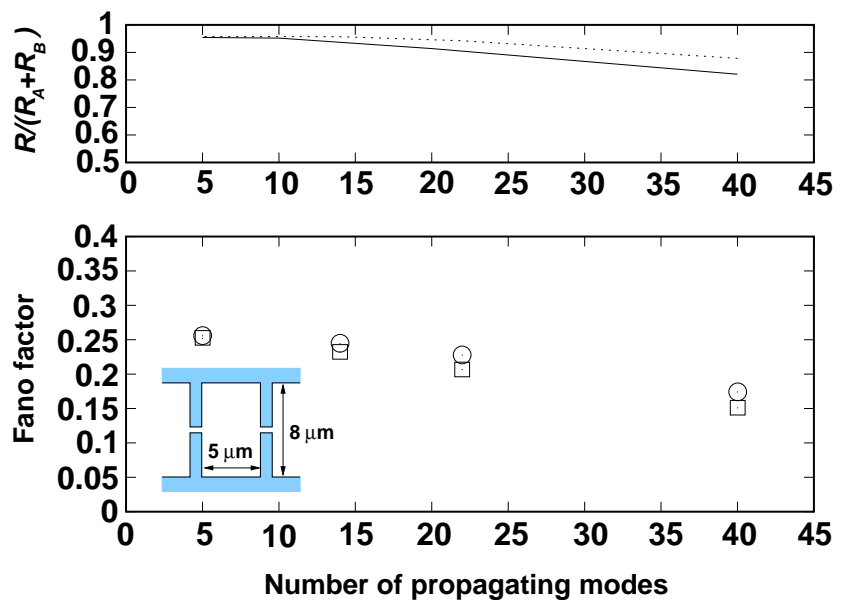

FIG. 1: Ratio of the cavity resistance to the sum of the constriction resistances $v s$. the number of modes propagating in the constrictions (upper panel): the solid line is for our rectangular cavity and the dotted line for a stadium-shaped cavity with a central constant-width region $1 \mu \mathrm{m}$ long. Numerical (empty circles) and experimental (empty squares, from Oberholzer et al. [13]) results for the Fano factor as a function of the number of modes propagating in the constrictions, for no applied magnetic field (lower panel). The inset contains a sketch of the model cavity.

cavity and the leads produce the mode mixing needed to generate a uniform occupancy of all the states at the same energy (i.e. the property defining an elastic quasireservoir), which, along with symmetry and integer transmission of the constrictions, represents the essential condition needed to achieve the $1 / 4$ suppression factor [15].

This is the reason why we prefer to define the subject of our investigation as "mesoscopic cavities," rather than "chaotic cavities," in order to avoid possible confusion with classical chaotic dynamics. Quantum diffraction occurring only at the input and output openings leads to multiple trajectories that create the equivalent of a chaotic behavior.

Our numerical technique is based on the evaluation of the transmission matrix of the structure being considered with the scattering matrix approach, which is relatively easy to implement and exhibits good numerical stability, also in the case of high values of the magnetic field $B$.

In particular, we consider a 2-dimensional Schrödinger equation in the $x-y$ plane, with $x$ being the direction of electron propagation (and thus $z$ the direction of the uniform orthogonal magnetic field). We choose a vector potential with a single nonzero component along the longitudinal direction $x$ (with a value $-B y$ ) and we subdivide the structure into a number of transverse slices, in each of which the scalar (confinement) potential can be assumed to be independent of $x$. In each slice we expand the transverse eigenfunctions onto a basis made up of the transverse eigenfunctions for $B=0$; the coefficients of this expansion and the associated longitudinal wave vectors are found solving an eigenvalue problem [16]. Then, with a mode-matching technique, we compute the scattering matrices of the sections extending from the middle of each slice to the middle of the following slice (and thus containing only one discontinuity of the potential). Composing the scattering matrices of all the sections [17], we find the S-matrix (and, as a submatrix, the transmission matrix $t$ with elements $t_{n m}$ ) of the overall structure.

The value of the conductance and of the shot noise power spectral density can be then computed by means of the relations [18]

$$
G=\frac{2 e^{2}}{h} \sum_{n, m}\left|t_{n m}\right|^{2}=\frac{2 e^{2}}{h} \sum_{j} w_{j}
$$

and

$$
S_{I}=4 \frac{e^{3}}{h}|V| \sum_{j} w_{j}\left(1-w_{j}\right)
$$

where the $w_{j}$ 's are the eigenvalues of the matrix $t t^{\dagger}, e$ is the electron charge, $h$ is Planck's constant, and $V$ is the externally applied voltage. Since the power spectral density of full shot noise is given by $S_{I}^{f s}=2 e|I|$ ( $I$ being the average current through the device) the Fano factor $\gamma$ can be written as

$$
\gamma=\frac{\sum_{j} w_{j}\left(1-w_{j}\right)}{\sum_{j} w_{j}}
$$

For a small number of modes propagating through the constrictions, quantum interference effects lead to wide relative fluctuations, as a function of energy, of both the numerator and the denominator of this expression. Averaging is therefore needed, and care must be taken to perform it correctly: in order to measure shot noise, the applied voltage $V$ must be much larger than $k T / e$ ( $k$ being the Boltzmann constant and $T$ the absolute temperature). Based on the detailed expression of the shot noise power spectral density at finite temperature provided by Büttiker [19], in the case of $e V \gg k T$ a good approximation of the shot noise term is given by a uniform average over an interval $e V$ around the Fermi level of eq. (2). An analogous conclusion, i.e., uniform averaging over the interval $e V$, can be drawn for the conductance term. We remark that it is essential that averaging be performed separately on the numerator and the denominator before taking the ratio (as in the actual measurement procedure).

Each of our data points has been obtained by averaging over the noise power spectral density and the conductance for 41 different energy values in an interval of width $0.22 \mathrm{meV}$ around the Fermi energy. In all of the following calculations we consider a Fermi energy $E_{f}$ of $9.134 \mathrm{meV}$ and the effective mass of gallium arsenide, $m^{*}=0.067 m_{0}$, with $m_{0}$ being the free electron mass (values consistent with the experiments by Oberholzer et al.).

This numerical approach has been used for the simulation of shot noise suppression in a rectangular cavity, first 

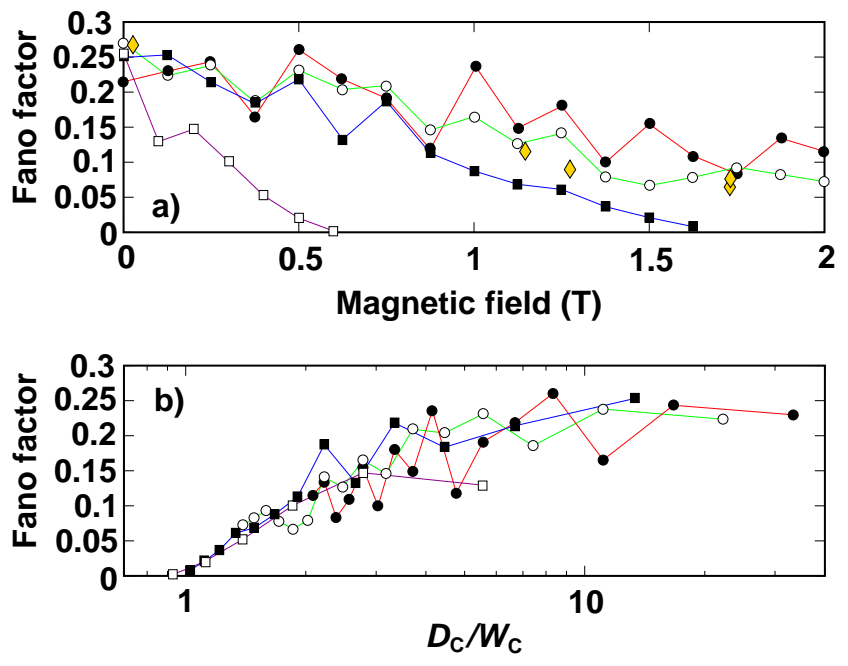

FIG. 2: (a) Fano factor as a function of the magnetic field computed for a mesoscopic cavity with constrictions of $40 \mathrm{~nm}$ (solid circles), $60 \mathrm{~nm}$ (empty circles), $100 \mathrm{~nm}$ (solid squares), $300 \mathrm{~nm}$ (empty squares). The diamonds represent the experimental data by Oberholzer et al.; (b) same data as in (a), but plotted as a function of the ratio of the classical cyclotron diameter $D_{C}$ to the constriction width $W_{C}$.

in the absence of a magnetic field. In particular, we show that the expected shot noise suppression is achieved with a simple hard-wall model defining a rectangular cavity, $5 \mu \mathrm{m}$ long and $8 \mu \mathrm{m}$ wide (corresponding to the lithographic dimensions of the cavity in refs. 7, 13]), with symmetric constrictions whose width is chosen on the basis of the desired number of propagating modes.

As constrictions are made wider (reaching a condition in which the analytical theory yielding the value $1 / 4$ for the suppression factor is not applicable any longer), we observe a decrease of the Fano factor, until it drops to zero as their width equals the cavity width and the structure becomes a noiseless perfect quantum wire. In the lower panel of fig. 11 we report the Fano factor from our numerical calculations (empty circles) as a function of the number of propagating modes: empty squares represent the experimental data obtained by Oberholzer et al. [13], and the agreement appears to be good, without the need for any fitting parameter. In the upper panel of fig. 11 we report the ratio of the overall cavity resistance to the sum of the constriction resistances, as a function of the number of propagating modes with a solid line for our rectangular cavity and with a dotted line for a stadium-shaped cavity $(8 \mu \mathrm{m}$ wide and $9 \mu \mathrm{m}$ long, since a horizontal stadium cannot be made with the same aspect ratio as that of the experimental cavity): such a quantity drops below unity, in disagreement with the experiment. We show also the calculation for the stadium-shaped cavity in order to exclude the possibility of a significant role played by a classically chaotic shape. Also the inclusion of disorder in the cavity (data for which are not reported here, due to space constraints) would not provide a valid justification of resistance behavior, since it would raise
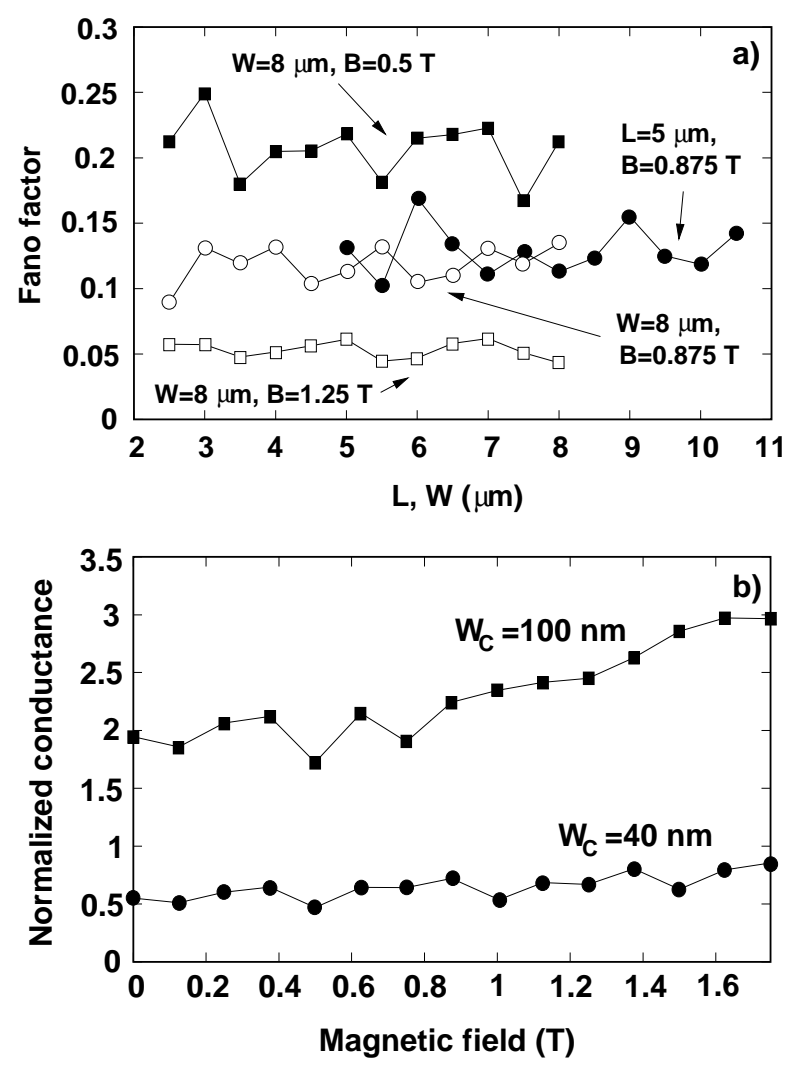

FIG. 3: (a) Fano factor as a function of the cavity width $W$ (solid circles) and length $L$ (remaining plots) for $100 \mathrm{~nm}$ symmetric constrictions; (b) conductance through the cavity, normalized with respect to the conductance quantum, as a function of the orthogonal magnetic field, for two values of the constriction widths.

both resistance and noise.

We suggest, as a possible explanation, that such an additivity over the whole measurement range can be the result of partial electron thermalization: as constrictions are made wider and electron diffraction is reduced, the total resistance of the cavity should start decreasing below the sum of the individual constrictions, according to the outcome of our ballistic calculation, while it would remain constant, independent of constriction width, if the cavity were a real reservoir, with electrons in thermal equilibrium with the lattice. If full thermalization of the electrons did occur, shot noise would also disappear (as long as the constrictions have integer transmission); a partial thermalization would instead determine an increase of the resistance toward the classical sum rule and, at the same time, a decrease of the noise, with respect to the ballistic result: this is consistent with the comparison between our results and those from the experiment.

We have then moved on to the simulation of the behavior of a cavity in the presence of a magnetic field orthogonal to the plane of the device, with a value up to a few tesla.

Results for the Fano factor are reported in fig. 2(a) 
for symmetric constrictions with a width of $40 \mathrm{~nm}$ (solid circles), $60 \mathrm{~nm}$ (empty circles), $100 \mathrm{~nm}$ (solid squares), and $300 \mathrm{~nm}$ (empty squares). The Fano factor exhibits a decrease as a function of the magnetic field, with a rate depending on the width of the constrictions and, therefore, on the number of modes propagating through such constrictions. The smaller the number of propagating modes the larger the fluctuations, due to the more significant relative contribution from quantum interference: for the narrowest constrictions residual fluctuations survive our averaging procedure.

The only available experimental data, from ref. [13], are for a single mode propagating through the constrictions [20], which corresponds, in our model, to a constriction width of $40 \mathrm{~nm}$. Experimental results from [13], represented with diamonds, lie slightly below the theoretical data for the $40 \mathrm{~nm}$ constrictions and close to those for the $60 \mathrm{~nm}$ constrictions. Thus our simulations tend to slightly overestimate the Fano factor, for narrow constrictions and large $B$ values. This can be attributed, in particular for a single propagating mode, to the specific scattering properties of hard-wall constrictions, which tend to create a set of modes inside the cavity, some of which are almost completely reflected at the exit constriction, contrary to gradual adiabatic constrictions (as we have verified with numerical simulations on a structure with openings defined as in ref. 21]).

In fig. 2(b) we report the same data as that of fig. 21(a), plotted as a function of the ratio of the classical cyclotron diameter $D_{C}$ (twice the cyclotron radius) to the constriction width $W_{C}$ : it is apparent that results for different constriction widths are essentially superimposed, thus demonstrating our conjecture that $D_{C} / W_{C}$ is the actually relevant parameter defining the level of shot noise suppression, simply because it determines the amount of scattering, and therefore diffraction, that occurs at the constrictions. Indeed, also the r.h.s. of eq. (4) of ref. [13] is proportional to $D_{C}$ and inversely proportional to $W_{C}$ : however it would also be proportional to the perimeter (or length), a dependence which is not observed in our results.

Running our simulations for different values of the cavity width and length (see fig. 3(a)), we have noticed that, in the regime of narrow constrictions and noise suppression due to the magnetic field, the Fano factor has only a mild and nonmonotonic dependence on such parameters (unless the constrictions are rather wide, and we are not in the regime of interest any longer), arguably resulting just from fluctuations due to interference effects. This is not in agreement with the interpretation of the phenomenon proposed by Oberholzer et al. 13]. Starting from a first-order expression involving the ratio of a quantum scattering time to the dwell time in the cavity, they suggest that the Fano factor should depend on the cavity area available for electron motion and they explain the shot noise suppression with a reduction of this area as a consequence of the formation of cyclotron orbits.

From the results of our numerical calculations, we propose a different and intuitive interpretation, directly based on the comparison of the cyclotron diameter with constriction width. In a semiclassical picture with skipping orbits crawling along the cavity walls, if the cyclotron diameter $D_{C}$ is much larger than the constriction width $W_{C}$, an electron impinging against such a constriction is likely to be reflected, thereby leading to diffraction. On the contrary, if $D_{C}$ is smaller than $W_{C}$, it is very likely that the electron traverses the two constrictions without undergoing reflections, so that shot noise is strongly suppressed (in the limit of no scattering, the Fano factor would drop to zero).

We have also computed the conductance of the cavity as a function of magnetic field, observing the transition, measured in ref. [13], between a condition, for $B=0$, in which it equals one half of the conductance of each constriction, and that for a large magnetic field, in which edge states are formed and it reaches the value of a single constriction. Results are reported in fig. 3(b), where the conductance for a constriction width of $40 \mathrm{~nm}$ (1 propagating mode) starts at 0.5 units and increases toward 1 unit as in the experimental results of ref. [13], while the curve for $W_{C}=100 \mathrm{~nm}$ (4 propagating modes for $B=0$ ) has an initial value of 2 and a value of 3 for a large magnetic field. This latter value is not 4 , as expected, because the total number of modes propagating through the constrictions decreases down to 3 at about $0.5 \mathrm{~T}$. We notice that the experimental data for conductance are in this case more closely reproduced by our ballistic simulation, as can be explained by the fact that in the presence of a magnetic field the effective area of the cavity and the time spent by each electron inside it are reduced, and therefore thermalization is decreased. We also wish to point out that, for the explanation of the observed phenomena, there is no need to invoke the presence of irregularities or defects in the cavity: indeed, as in the case of no magnetic field, they would lead to an increase of noise, due to the resulting electron diffraction.

In conclusion, we have presented a numerical simulation of shot noise suppression in mesoscopic cavities, reproducing the results of recent experiments without the need for fitting parameters. In particular, we get very good agreement with the measured behavior of the Fano factor as a function of the width of the constrictions and, although with fluctuations due to interference effects, we are able to closely reproduce the dependence of the Fano factor on magnetic field. From our results, we observe that the reduction of the Fano factor with increasing magnetic field can be simply explained as the consequence of the decrease in the ratio of the cyclotron diameter to the constriction width, which leads to progressively improved transmission through the apertures, and therefore suppression of diffraction.

This work has been supported by the Italian Ministry for Education, University and Research, through the FIRB project "Nanotechnologies and nanodevices for the information society" and through the PRIN "Excess noise in nanoscale devices." 
[1] Beenakker C. W. J. and Büttiker M., Phys. Rev. B, 46 (1992) 1889.

[2] Jalabert R. A., Pichard J. L. and Beenakker C. W. J., Europhys. Lett., 27 (1994) 255.

[3] Beenakker C. W. J., Phys. Rev. Lett., 82 (1999) 2761.

[4] Agam O., Aleiner I. and Larkin A., Phys. Rev. Lett., 85 (2000) 3153.

[5] Silvestrov P. G., Goorden M. C. and Beenakker C. W. J., Phys. Rev. B, 67 (2003) 241301(R).

[6] Henny M., Oberholzer S., Strunk C. and Schönenberger C., Phys. Rev. B, 59 (1999) 2871.

[7] Oberholzer S., Sukhorukov E. V., Strunk C., Schönenberger C., Heinzel T. and Holland M., Phys. Rev. Lett., 86 (2001) 2114.

[8] Beenakker C. W. J., Rev. Mod. Phys., 69 (1997) 731.

[9] Blanter Ya. M. and Sukhorukov E. V., Phys. Rev. Lett., 84 (2000) 1280.

[10] van Langen S. A. and Büttiker M., Phys. Rev. B, 56
(1997) R1680.

[11] Polianski M. L., Samuelsson P. and Büttiker M., Phys. Rev. B, 72 (2005) 161302(R).

[12] Blanter Ya. M., Büttiker M., Phys. Rep., 336 (2000) 1.

[13] Oberholzer S., Sukhorukov E. V. and Schönenberger C., Nature, 415 (2002) 765.

[14] Aigner F., Rotter S. and Burgdörfer J., Phys. Rev. Lett., 94 (2005) 216801.

[15] Oberholzer S., Sukhorukov E. V., Strunk C. and Schonenberger C., Phys. Rev. B., 66 (2002) 233304.

[16] Tamura H. and Ando T., Phys. Rev. B, 44 (1991) 1792.

[17] Datta S., Electronic Transport in Mesoscopic Systems (Cambridge University Press, Cambridge) 1995, p. 125.

[18] Büttiker M., Phys. Rev. Lett., 65 (1990) 2901.

[19] Büttiker M., Phys. Rev. B, 46 (1992) 12485.

[20] Oberholzer S., private communication

[21] Ando T., Phys. Rev. B, 44 (1991) 8017. 